\title{
Sensitivity filtering from the non-local perspective
}

\section{Evgrafov, Anton; Bellido, José C.}

\section{Published in:}

Structural and Multidisciplinary Optimization

Link to article, DOI:

10.1007/s00158-019-02303-w

Publication date:

2019

Document Version

Peer reviewed version

Link back to DTU Orbit

\section{Citation (APA):}

Evgrafov, A., \& Bellido, J. C. (2019). Sensitivity filtering from the non-local perspective. Structural and Multidisciplinary Optimization, 60(1), 401-404 . https://doi.org/10.1007/s00158-019-02303-w

\section{General rights}

Copyright and moral rights for the publications made accessible in the public portal are retained by the authors and/or other copyright owners and it is a condition of accessing publications that users recognise and abide by the legal requirements associated with these rights.

- Users may download and print one copy of any publication from the public portal for the purpose of private study or research.

- You may not further distribute the material or use it for any profit-making activity or commercial gain

- You may freely distribute the URL identifying the publication in the public portal

If you believe that this document breaches copyright please contact us providing details, and we will remove access to the work immediately and investigate your claim. 


\title{
Sensitivity filtering from the non-local perspective
}

\author{
Anton Evgrafov • José C. Bellido
}

Received: date / Accepted: date

\begin{abstract}
We present a mathematical model of topology optimization for non-local linear diffusion equation. The model provides a rigorous and constructive answer to the challenge of interpreting sensitivity filtering as an internal feature of a specific continuum mechanics model, which has been raised in Sigmund O., Maute K.: Sensitivity filtering from a continuum mechanics perspective. Struct. Multidiscip. O. 46(4),471-475 (2012). Our model enjoys two very interesting properties. On the one hand, the governing non-local integral equations of this model approximate the well-known local generalized Laplace equation with diffusion coefficient obeying SIMP (Solid Isotropic Material with Penalization) law. On the other hand, the topology optimization problem admits optimal solutions for a range of penalization parameters of practical interest without the need for any external regularization techniques, something which is well known to be false in the case of topology optimization with SIMP for classical local description of continuum mechanics.
\end{abstract}

A.E.'s research is funded by the Villum Fonden through the Villum Investigator Project InnoTop. The work of J.C.B. is funded by FEDER EU and Ministerio de Economía y Competitividad (Spain) through grant MTM2017-83740-P. The authors declare that they have no conflict of interest.

\footnotetext{
A. Evgrafov

Department of Mechanical Engineering

Technical University of Denmark

DK-2800 Kgs Lyngby, Denmark

Tel.: +4545254205

E-mail: aaev@mek.dtu.dk

J.C. Bellido

Department of Mathematics

University of Castilla-La Mancha

13.071 Ciudad Real, Spain

Tel.: +34 926295261

E-mail: JoseCarlos.Bellido@uclm.es
}

Keywords non-local optimal design - Existence of solutions · SIMP · Peridynamics

\author{
Mathematics Subject Classification \\ (2010) 45A05 · 49J45 · 49K20 · 49K21 · 80M50
}

\section{Introduction}

Sigmund and Maute (2012) have raised a curious research question: can the sensitivity filtering, which has been utilized as a heuristic regularization scheme in the earliest work on compliance minimization with SIMP model (see Bendøse and Sigmund, 2003; Allaire, 2012, and references therein), be viewed as a natural byproduct of applying homogenization based topology optimization to a non-local mechanical model? Whereas the answer provided in (Sigmund and Maute, 2012) is unsatisfactory, the question has generated enough interest that other researchers (see for example Li et al., 2017; Bellido, 2015) followed the suit. Yet to this point a plausible interpretation of sensitivity filtering has remained elusive. Correcting this state of affairs is the main objective of the present brief note.

We build upon the recent work on non-local optimal design by Andrés and Muñoz (2015), which utilizes an integral, derivative-free description of continuum mechanics (Kunin, 1975; Silling, 2000). Our optimization problem will employ a non-local linear diffusion model, which can represent a broad range of physical phenomena such as the steady state heat conduction, Darcy flow through the porous medium, or small deflection of an elastic membrane; generalizing our results to linear elasticity involving vector fields is high on our research agenda. We will stick to the vocabulary of heat conduction henceforth referring to the design parame- 
ters as conductivities and to the corresponding states as temperatures.

\section{The local problem}

We set the stage for a forthcoming discussion by recollecting the classical compliance minimization problem with SIMP model for the generalized Laplace equation (by our convention modelling the steady state heat conduction). Let $\Omega \subset \mathbb{R}^{n}, n \in \mathbb{N}$ be an open bounded domain with Lipschitz continuous boundary. In this domain we would like to distribute isotropic heterogeneous heat conducting material with conductivity $\kappa^{\text {loc }}$ satisfying the SIMP model $\kappa^{\text {loc }}(x)=\rho^{p}(x), x \in \Omega$, where $p \geq 1$ is a penalty parameter, and $\rho$ is a design variable taken from the admissible set

$\mathcal{A}^{\mathrm{loc}}=\left\{\rho \in L^{\infty}(\Omega)\left|\rho(\cdot) \in[\underline{\rho}, \bar{\rho}], \int_{\Omega} \rho(x) \mathrm{d} x=\gamma\right| \Omega \mid\right\}$.

We will assume that the bounds satisfy the inequalities $0<\rho<\bar{\rho}<+\infty$, and that the volume fraction $\gamma \in] \underline{\rho}, \bar{\rho}\left[\overline{\text { to }}\right.$ avoid trivial situations. Let $f \in L^{2}(\Omega)$ be the volumetric heat source applied in $\Omega$. It is well known that the steady state temperature in $\Omega$, assuming homogeneous Dirichlet boundary conditions, is the unique $u \in \mathcal{U}_{0}^{\text {loc }}=H_{0}^{1}(\Omega)$, which minimizes the quadratic energy functional

$v \mapsto I_{\kappa^{\mathrm{loc}}}^{\mathrm{loc}}(v):=\frac{1}{2} \int_{\Omega} \kappa^{\mathrm{loc}}|\nabla v|^{2} \mathrm{~d} x-\int_{\Omega} f v \mathrm{~d} x$.

The compliance minimization problem in this notation can be stated as a saddle-point problem

$\max _{\rho \in \mathcal{A}^{\mathrm{loc}}} \min _{u \in \mathcal{U}_{0}^{\mathrm{loc}}} I_{\rho^{p}}^{\mathrm{loc}}(u)$

This problem famously attains its maximum for $p=1$ (convex case), and does not attain its maximum for $p>1$, see (Allaire, 2012; Bendøse and Sigmund, 2003; Céa and Malanowski, 1970).

Let us denote by $\rho \mapsto c_{\mathrm{loc}}(\rho):=-2 I_{\rho^{p}}^{\mathrm{loc}}\left(u_{\rho}\right)$ the (scaled) reduced objective function of (2), where $u_{\rho}$ is the steady state temperature corresponding to the material distribution $\rho \in \mathcal{A}^{\text {loc }}$. The first variation of this function in the direction $\xi \in L^{\infty}(\Omega)$ is given by the formula

$c_{\mathrm{loc}}^{\prime}(\rho ; \xi)=-p \int_{\Omega} \rho^{p-1}(x) \xi(x)\left|\nabla u_{\rho}(x)\right|^{2} \mathrm{~d} x$,

and therefore the function $\nabla_{\rho} c_{\text {loc }}=-p \rho^{p-1}\left|\nabla u_{\rho}\right|^{2}$ may be thought of as the gradient of the (reduced) compliance. "Sensitivity filtering" can be viewed as regularizing the derivatives $c_{\text {loc }}^{\prime}$ by taking a convolution of the underlying gradient $\nabla_{\rho} c_{\text {loc }}$ with a suitable
Lipschitz continuous radial kernel $A_{\delta}^{\text {loc }}: \mathbb{R}_{+} \rightarrow \mathbb{R}_{+}$ with a small compact support (often $A_{\delta}^{\text {loc }}\left(\left|x-x^{\prime}\right|\right):=$ $c_{\text {nrm }} \max \left\{\delta^{\text {loc }}-\left|x-x^{\prime}\right|, 0\right\}$, for some $\delta^{\text {loc }}>0$ and a normalization constant $c_{\mathrm{nrm}}>0$ ), that is,

$$
\begin{aligned}
& \tilde{c}_{\mathrm{loc}}^{\prime}(\rho ; \xi)=-p \int_{\Omega} \xi(x) \times \\
& \times \int_{\Omega} A_{\delta}^{\mathrm{loc}}\left(\left|x-x^{\prime}\right|\right) \underbrace{\rho^{p-1}\left(x^{\prime}\right)\left|\nabla u_{\rho}\left(x^{\prime}\right)\right|^{2}}_{=-\nabla_{\rho} c_{\mathrm{loc}}\left(x^{\prime}\right) / p} \mathrm{~d} x^{\prime} \mathrm{d} x .
\end{aligned}
$$

Interestingly enough, there is convincing numerical evidence that the variational inequality $\tilde{c}_{\text {loc }}^{\prime}\left(\rho^{*} ; \rho-\rho^{*}\right) \geq 0$, $\forall \rho \in \mathcal{A}^{\text {loc }}$, which is a perturbed version of first order necessary optimality conditions for (2), admits solutions $\rho^{*} \in \mathcal{A}^{\mathrm{loc}}$, and these solutions are of practical value for the users of topology optimization method.

\section{The non-local problem}

The non-local model of steady state heat conduction that we consider can be conveniently obtained by replacing the quadratic energy functional (1) containing first order derivatives of the temperature with a non-local (double integral) quadratic functional, which only involves function values. More precisely, let $\delta>0$ be the non-local interaction horizon; only the points which are closer than $\delta$ can directly interact with each other. Let $\Omega_{\delta}=\cup_{x \in \Omega} B(x, \delta)$, where $B(x, \delta)=\left\{x^{\prime} \in\right.$ $\left.\mathbb{R}^{n}:\left|x-x^{\prime}\right|<\delta\right\} . \Omega_{\delta}$ is therefore the set of points, which points in $\Omega$ can interact with, and we denote by $\Gamma^{\mathrm{nl}}=\Omega_{\delta} \backslash \Omega$ the "non-local boundary" of $\Omega$. In the model we consider interaction of two points $x, x^{\prime} \in \Omega_{\delta}$ with temperatures $u(x)$ and $u\left(x^{\prime}\right)$ results in the bond energy density of

$i_{\delta, \kappa}(u):=\kappa\left(x, x^{\prime}\right) A_{\delta}\left(\left|x-x^{\prime}\right|\right) \frac{\left(u(x)-u\left(x^{\prime}\right)\right)^{2}}{\left|x-x^{\prime}\right|^{2}}$,

where $\kappa: \Omega_{\delta}^{2} \rightarrow[\underline{\kappa}, \bar{\kappa}] \subset \mathbb{R}_{++}$is the non-local conductivity, and $A_{\delta}: \mathbb{R}_{+} \rightarrow \mathbb{R}_{+}$is a non-negative radial function, which vanishes for $\left|x-x^{\prime}\right| \geq \delta$ (points outside the horizon do not interact) and behaves like $\left|x-x^{\prime}\right|^{-(n+2 s-2)}, 0<s<1$, for small $\left|x-x^{\prime}\right|$. The non-local Dirichlet principle is that the steady state of this thermal system is the state of the minimal energy as defined by the following quadratic energy functional

$v \mapsto I_{\delta, \kappa}(v):=\frac{1}{2} \int_{\Omega_{\delta}} \int_{\Omega_{\delta}} i_{\delta, \kappa}(v) \mathrm{d} x \mathrm{~d} x^{\prime}-\int_{\Omega} f v \mathrm{~d} x$

The minimum of $I_{\delta, \kappa}(u)$ is attained when taken over the natural energy space $\mathcal{U}_{0} \subset L^{2}\left(\Omega_{\delta}\right)$ of functions satisfying the non-local homogeneous Dirichlet boundary conditions $\left.u\right|_{\Gamma^{\mathrm{nl}}}=0$ and having the finite energy 

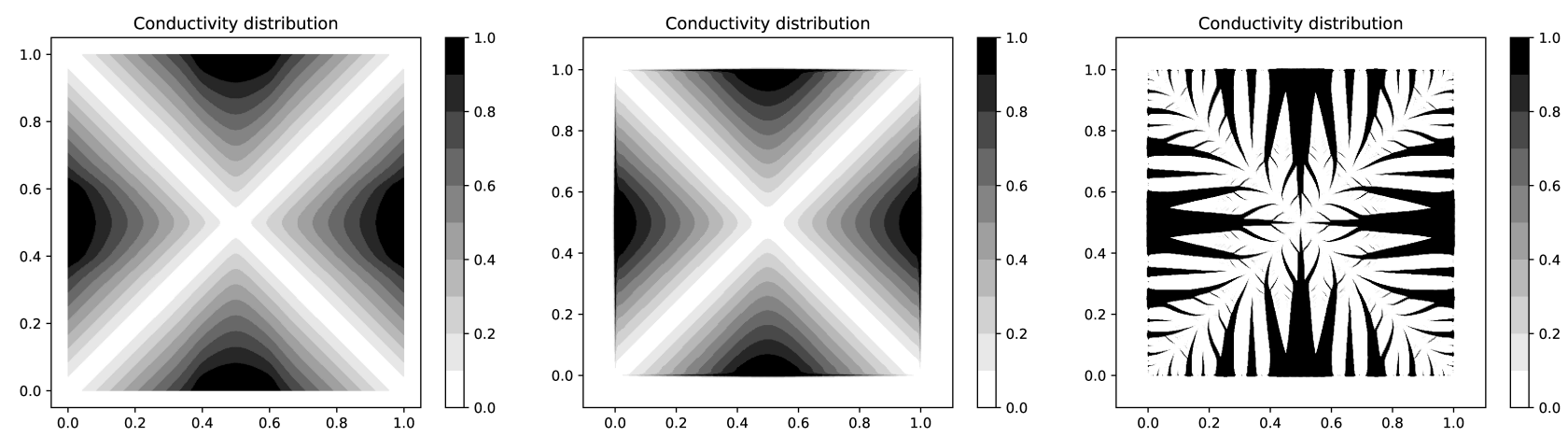

Fig. 1 Left: optimal design for the local problem (2) corresponding to $p=1$. Middle: optimal design for the non-local problem (6) corresponding to $p=1$. Right: locally optimal (the problem is not convex) design for the non-local problem (6) corresponding to $p=2$. In all figures, $\kappa^{\text {loc }}=\rho^{p}$ is plotted.

$\int_{\Omega_{\delta}} \int_{\Omega_{\delta}} i_{\delta, \kappa}(u) \mathrm{d} x \mathrm{~d} x^{\prime}<+\infty$; see for example (Bellido and Mora-Corral, 2014) for more details and generalizations.

We now assume that the non-local conductivity is a geometric mean of local conductivities obeying SIMP, that is $\kappa\left(x, x^{\prime}\right)=\sqrt{\kappa^{\operatorname{loc}}(x) \kappa^{\operatorname{loc}}\left(x^{\prime}\right)}=\rho^{p / 2}(x) \rho^{p / 2}\left(x^{\prime}\right)$. Similarly to $\mathcal{A}^{\text {loc }}$, we define the admissible set for the non-local problem by

$\mathcal{A}=\left\{\rho \in L^{\infty}\left(\Omega_{\delta}\right)\left|\rho(\cdot) \in[\underline{\rho}, \bar{\rho}], \int_{\Omega_{\delta}} \rho(x) \mathrm{d} x=\gamma\right| \Omega \mid\right\}$,

where the volume fraction $\gamma \in] \rho\left|\Omega_{\delta}\right| /|\Omega|, \bar{\rho}\left|\Omega_{\delta}\right| /|\Omega|[$ to avoid trivial cases. Also, similarly to (2) we define the non-local compliance minimization problem as the following saddle point problem:

$\max _{\rho \in \mathcal{A}} \min _{u \in \mathcal{U}_{0}} I_{\delta, \rho^{p / 2}(x) \rho^{p / 2}\left(x^{\prime}\right)}(u)$.

\section{Discussion of the non-local problem}

Existence of optimal solutions: Recall that the local compliance minimization problem (2) admits optimal solutions in the convex case corresponding to $p=1$, and lacks optimal solutions (without further regularization) in the non-convex case $p>1$, see (Allaire, 2012; Bendøse and Sigmund, 2003; Céa and Malanowski, 1970). The non-local compliance minimization problem (6) behaves in a remarkably different fashion.

Theorem 1 For each $p \in[1,2]$ the problem (6) admits an optimal solution.

Proof We note that the mapping $L^{\infty}\left(\Omega_{\delta} \times \Omega_{\delta}\right) \ni \kappa \mapsto$ $I_{\delta, \kappa^{p / 2}}(u) \in \mathbb{R}$ is concave and continuous (for $0<\underline{\kappa} \leq$ $\kappa \leq \bar{\kappa}<\infty)$ for each $p \in[1,2]$ and $u \in \mathcal{U}_{0}$, whence also weak* sequentially upper semicontinuous. The latter also holds true for the function $\kappa \mapsto \min _{u \in \mathcal{U}_{0}} I_{\delta, \kappa^{p / 2}}(u)$ because the upper-semicontinuity is preserved under taking the infimum. Additionally, the map $\mathcal{A} \ni \rho \mapsto$ $\rho(x) \rho\left(x^{\prime}\right) \in L^{\infty}\left(\Omega_{\delta} \times \Omega_{\delta}\right)$ maps weak* converging sequences into weak* converging sequences. It remains to note that the set $\mathcal{A}$ is non-empty, bounded, closed, and convex in $L^{\infty}\left(\Omega_{\delta}\right)$ (hence also weak* sequentially compact), therefore the generalized Weierstrass' theorem is applicable.

Relation to "sensitivity filtering": We now return to the issue of the sensitivity filtering raised in (Sigmund and Maute, 2012). Indeed, taking the variation of the compliance functional $c(\rho)=-2 I_{\delta, \rho^{p / 2}(x) \rho^{p / 2}\left(x^{\prime}\right)}\left(u_{\rho}\right)$ at a point $\rho \in \mathcal{A}$ in some direction $\xi \in L^{\infty}\left(\Omega_{\delta}\right)$, we obtain the expression

$$
\begin{array}{r}
c^{\prime}(\rho ; \xi)=-\frac{p}{2} \int_{\Omega_{\delta}} \int_{\Omega_{\delta}}\left[\rho^{p / 2-1}(x) \rho^{p / 2}\left(x^{\prime}\right) \xi(x)\right. \\
\left.+\rho^{p / 2}(x) \rho^{p / 2-1}\left(x^{\prime}\right) \xi\left(x^{\prime}\right)\right] \times \\
\times A_{\delta}\left(\left|x-x^{\prime}\right|\right) \frac{\left(u_{\rho}(x)-u_{\rho}\left(x^{\prime}\right)\right)^{2}}{\left|x-x^{\prime}\right|^{2}} \mathrm{~d} x^{\prime} \mathrm{d} x,
\end{array}
$$

where $u_{\rho} \in \mathcal{U}_{0}$ is the steady state temperature corresponding to the material distribution $\rho \in \mathcal{A}$. In particular, for the interesting case $p=2$ we obtain (note the striking similarity with (3) for $p=2$ )

$$
\begin{aligned}
& c^{\prime}(\rho ; \xi)=-2 \int_{\Omega_{\delta}} \xi(x) \times \\
& \times \underbrace{\int_{\Omega_{\delta}} A_{\delta}\left(\left|x-x^{\prime}\right|\right) \rho\left(x^{\prime}\right) \frac{\left(u_{\rho}(x)-u_{\rho}\left(x^{\prime}\right)\right)^{2}}{\left|x-x^{\prime}\right|^{2}} \mathrm{~d} x^{\prime}}_{=-\nabla_{\rho} c(x) / 2} \mathrm{~d} x,
\end{aligned}
$$

and therefore we can interpret the underlined expression (up to the scaling factor of $-1 / 2$ ) as the gradient of the compliance. Note that this expression can be viewed as a weighed non-local average of a local bond energy density, precisely as desired in Sigmund and Maute (2012). It should also be noted that 
$\nabla_{\rho} c(x) \approx-2 \rho(x)\left|\nabla u_{\rho}(x)\right|^{2}=\nabla_{\rho} c_{\text {loc }}(x)$ under appropriate assumptions on $u_{\rho}$ and $\rho$ for small $\delta$, see Ponce (2004), thus the "filtering" disappears in the limit of small horizons and only the standard gradient of the local compliance remains.

We would like to remark that the multiplicative structure of the non-local conductivity model is crucial both for obtaining existence of optimal solutions for $p \in(1,2]$ and for recovering the the sensitivity filtering from the non-local model. None of these features hold if one considers the additive parametrization proposed in Andrés and Muñoz (2015).

Relation to the local problem: The local compliance optimization problem can be recovered from the the nonlocal compliance problems in the limit of vanishing interaction horizons, in the sense that minimizers of the non-local problem converge to minimizers of the local one as $\delta \rightarrow 0$. This was shown for a "SIMP-less" parametrization model in Andrés and Muñoz (2015), and we plan to address this question for our model in the near future.

\section{Numerical example}

For illustrative purposes we include a numerical example, where we use a uniform heat source $f \equiv 1, n=2$, $\Omega=] 0,1\left[2, \delta=0.05, s=1 / 3, \gamma=0.4, \rho=10^{-3}\right.$, $\bar{\rho}=1.0$. The problem is solved on a regular grid, where $\Omega$ is decomposed into $320^{2}$ squares, and each square is split into two triangles. Standard piecewise-linear continuous Lagrange elements are used for approximating functions in $\mathcal{U}_{0}$, and piecewise-constants for approximating functions in $\mathcal{A}$. Galerkin-Riesz method is used for discretizing the non-local state equations corresponding to minimizing the quadratic energy functional (5). The resulting optimal solutions to the nonlocal and local problems (6), (2) are shown in Fig. 1.

\section{Concluding remarks}

We have presented a brief introduction of a nonlocal topology optimization model which, unlike its local counterpart, admits globally optimal solutions for the compliance minimization problem with SIMP for penalty values $p \in[1,2]$. The gradient of the compliance in this framework has a structure, which bears a striking resemblance to "sensitivity filtering" thus addressing the issue raised in Sigmund and Maute (2012). In this fashion we have addressed not one but two important open research questions in the topology optimization literature.

\section{Replication of results}

The format of a brief note does not allow us to go into details of the numerical results, included as an illustration of our work. We are currently preparing a manuscript dedicated to a significantly more detailed exploration of the presented non-local topology optimization model from both the theoretical and the numerical angles.

\section{References}

Allaire G (2012) Shape optimization by the homogenization method, vol 146. Springer Science \& Business Media

Andrés F, Muñoz J (2015) Nonlocal optimal design: a new perspective about the approximation of solutions in optimal design. Journal of Mathematical Analysis and Applications 429(1):288-310

Bellido JC (2015) Peridynamics and topology optimization, 11th World Congress on Structural and Multidisciplinary Optimisation, 07-12 June 2015, Sydney, Australia

Bellido JC, Mora-Corral C (2014) Existence for nonlocal variational problems in peridynamics. SIAM J Math Anal 46(1):890-916, DOI 10.1137/130911548

Bendøse M, Sigmund O (2003) Topology Optimization: Theory, Methods and Applications. ISBN: 3540-42992-1. Springer

Céa J, Malanowski K (1970) An example of a maxmin problem in partial differential equations. SIAM Journal on Control 8(3):305-316

Kunin I (1975) Теория упругих сред с микроструктурой: нелокальная теория упругости (Theory of elastic bodies with microstructure: nonlocal theory of elasticity). Издательство Наука (Izd Nauka), Moscow (in Russian)

Li L, Zhang G, Khandelwal K (2017) Topology optimization of structures with gradient elastic material. Structural and Multidisciplinary Optimization 56(2):371-390

Ponce AC (2004) An estimate in the spirit of Poincaré's inequality. Journal of the European Mathematical Society 6(1):1-15

Sigmund O, Maute K (2012) Sensitivity filtering from a continuum mechanics perspective. Structural and Multidisciplinary Optimization 46(4):471-475

Silling SA (2000) Reformulation of elasticity theory for discontinuities and long-range forces. Journal of the Mechanics and Physics of Solids 48(1):175-209 